\title{
CR2 Gene
}

National Cancer Institute

\section{Source}

National Cancer Institute. CR2 Gene. NCI Thesaurus. Code C104977.

This gene plays a role in the innate immune response. 